\title{
Prostate Health Index and Prostate Health Index Density as Diagnostic Tools for Improved Prostate Cancer Detection
}

\author{
Marija Barisiene ${ }^{D},{ }^{1}$ Arnas Bakavicius, ${ }^{1}$ Diana Stanciute, ${ }^{2,3}$ Jolita Jurkeviciene, ${ }^{4}$ \\ Arunas Zelvys, ${ }^{1}$ Albertas Ulys, ${ }^{1}$ Dalius Vitkus $\mathbb{D}^{\mathbb{D}},{ }^{1}$ and Feliksas Jankevicius ${ }^{1}$ \\ ${ }^{1}$ Institute of Clinical Medicine, Faculty of Medicine, Vilnius University, Vilnius 03101, Lithuania \\ ${ }^{2}$ Laboratory of Molecular Oncology, National Cancer Institute, Vilnius 08660, Lithuania \\ ${ }^{3}$ Faculty of Health Sciences, Klaipeda University, Klaipeda 92294, Lithuania \\ ${ }^{4}$ Centre of Laboratory Medicine, Vilnius University Hospital Santaros Klinikos, Vilnius 08661, Lithuania
}

Correspondence should be addressed to Marija Barisiene; m.barisiene@gmail.com

Received 2 January 2020; Revised 2 June 2020; Accepted 29 June 2020; Published 22 July 2020

Academic Editor: Cristiana Corsi

Copyright $\odot 2020$ Marija Barisiene et al. This is an open access article distributed under the Creative Commons Attribution License, which permits unrestricted use, distribution, and reproduction in any medium, provided the original work is properly cited.

Background. To evaluate the diagnostic potential of [-2] proPSA (p2PSA), \%p2PSA, Prostate Health Index (phi), and phi density (PHID) as independent biomarkers and in composition of multivariable models in predicting high-grade prostatic intraepithelial neoplasia (HGPIN) and overall and clinically significant prostate cancer (PCa). Methods. 210 males scheduled for prostate biopsy with total PSA (tPSA) range $2-10 \mathrm{ng} / \mathrm{mL}$ and normal digital rectal examination were enrolled in the prospective study. Blood samples to measure tPSA, free PSA (fPSA), and p2PSA were collected immediately before 12-core prostate biopsy. Clinically significant PCa definition was based on Epstein's criteria or ISUP grade $\geq 2$ at biopsy. Results. PCa has been diagnosed in 112 $(53.3 \%)$ patients. Epstein significant and ISUP grade $\geq 2$ PCa have been identified in 81 (72.3\%) and 40 (35.7\%) patients, respectively. Isolated HGPIN at biopsy have been identified in $24(11.4 \%)$ patients. Higher p2PSA and its derivative mean values were associated with PCa. At $90 \%$ sensitivity, PHID with cut-off value of 0.54 have demonstrated the highest sensitivity of $35.7 \%$ for overall PCa detection, so PHID and phi with cut-off values of 33.2 and 0.63 have demonstrated the specificity of $34.7 \%$ and $34.1 \%$ for ISUP grade $\geq 2$ PCa detection at biopsy, respectively. In univariate ROC analysis, PHID with AUC of 0.77 and 0.80 was the most accurate predictor of overall and Epstein significant PCa, respectively, so phi with AUC of 0.77 was the most accurate predictor of ISUP grade $\geq 2 \mathrm{PCa}$ at biopsy. In multivariate logistic regression analysis, phi improved diagnostic accuracy of multivariable models by $5 \%$ in predicting ISUP grade $\geq 2$ PCa. Conclusions. PHID and phi have shown the greatest specificity at $90 \%$ sensitivity in predicting overall and clinically significant $\mathrm{PCa}$ and would lead to significantly avoid unnecessary biopsies. PHID is the most accurate predictor of overall and Epstein significant PCa, so phi is the most accurate predictor of ISUP grade $\geq 2$ PCa. phi significantly improves the diagnostic accuracy of multivariable models in predicting ISUP grade $\geq 2 \mathrm{PCa}$.

\section{Introduction}

Prostate cancer (PCa) is the second most common cancer in males worldwide accounting for $13.5 \%$ of all new cancer cases and the sixth leading cause of cancer-related death among males [1]. Australia and New Zealand, so the Northern and Western European countries, belong to the highest incidence rate regions in the world (age standardized rate (ASR) per 100,000 of $86.4,85.7$, and 75.8 , respectively), largely due to widespread use of prostate-specific antigen
(PSA) testing and subsequent prostate biopsies (PB) $[1,2]$. $\mathrm{PCa}$ is the 3rd most commonly diagnosed cancer by cancer site and the most common cancer among males in Lithuania with ASR of 70.2 per 100,000 males. It is the third most common cancer-related death among Lithuanian males (ASR: 11.3 per 100,000$)$ [1]

Due to low PSA specificity in determining PCa, especially with the total PSA (tPSA) level below $10 \mathrm{ng} / \mathrm{mL}$, the risk of PCa in males with tPSA between 4.1 and $9.9 \mathrm{ng} / \mathrm{mL}$ and negative digital rectal examination (DRE) is about $20 \%$ with $85 \%$ 
probability, respectively, that these cancers would be organ confined [3]. On the other hand, some males may harbor PCa despite very low tPSA levels $(<2.0 \mathrm{ng} / \mathrm{mL})$ [4]. In up to $25 \%$ of all $\mathrm{PB}$, premalignant condition, namely, high-grade prostatic intraepithelial neoplasia (HGPIN), is diagnosed [5, 6]. Of all screen-detected PCa based on tPSA screening programs, $42 \%$ are clinically insignificant, which means that the disease would never lead to clinical symptoms or death [7]. In turn, detection of clinically insignificant PCa consequently leads to overtreatment with its potential side effect profile or may cause an anxiety related to active surveillance strategy, so deterioration of quality of life in any scenario is inevitable in aging society. Thus, the decision to perform a PB with intent to detect clinically significant $\mathrm{PCa}$, which indeed needs treatment, in males with tPSA levels within the "grey" zone is one of the main concerns in daily practice.

To overcome the limitations of tPSA, p2PSA derivatives, such as Prostate Health Index (phi) and percentage of p2PSA (\%p2PSA), which combine the results of quantitative kallikrein immunoassays into a single numerical score, have been suggested. \%p2PSA and phi have been associated with improved overall and aggressive PCa detection over tPSA and $\mathrm{PPSA} / \mathrm{tPSA}$ ratio (\%fPSA) in several studies [8-11]. The objective of the present study was to evaluate the diagnostic potential of p2PSA, \%p2PSA, phi, and phi density (PHID) as independent biomarkers and in combination with other demographic and clinical parameters, to predict overall and clinically significant PCa. The ability of p2PSA and its derivatives to discriminate HGPIN at biopsy was evaluated.

\section{Materials and Methods}

2.1. Patients and Samples. A prospective clinical trial was initiated at Vilnius University Hospital Santaros Klinikos (Lithuania) and National Cancer Institute (Lithuania) from January 2015 till December 2016. Males older than 50 years old with tPSA range from 2 to $10 \mathrm{ng} / \mathrm{mL}$ and normal DRE referred for $\mathrm{PB}$ were included into the study. Previous diagnosis of $\mathrm{PCa}$, history of open or endoscopic prostate surgery, $\mathrm{PB}$ within 3 months prior the study, usage of 5-alphareductase inhibitors, active urinary tract infection, and acute prostatitis were considered exclusion criteria. The study was approved by the Regional Biomedical Research Ethics Committee (Nr 158200-14-759-273), and written informed consent was obtained from all patients.

Blood samples to measure tPSA, fPSA, and p2PSA were collected immediately before $\mathrm{PB}$ for every patient and processed within 3 hours after the collection due to instability of p2PSA at room temperature [12]. The samples were stored at $-80^{\circ} \mathrm{C}$ before testing in a single laboratory using the Beckman Coulter Access ${ }^{\circledR} 2$ Immunoassay Analyzer and Access Hybritech $^{\circledR}$ reagents and calibrators for all assays, including tPSA, fPSA, and p2PSA. Hybritech calibration was used for tPSA and fPSA. \%p2PSA was calculated using formula (p2 PSA/free PSA $($ fPSA $)) \times 100$ and phi as $($ p2PSA/fPSA $) \times \sqrt{ }$ tPSA [13].

Transrectal ultrasound was used to measure prostate. Prostate volume (PV) was calculated using formula prostate length $\times$ height $\times$ width $\times 0.52$. PSA density (PSAD) and
PHID were calculated as TPSA and phi divided by the volume of the prostate. $\mathrm{PB}$ was performed by transrectal approach using the standardized 12-core random sampling protocol. PB specimens were evaluated at National Centre of Pathology (Lithuania) by dedicated pathologists blinded to the blood serum results. Gleason score was evaluated according to the 2005 Guidelines of International Society of Urological Pathology (ISUP), and ISUP grades were assigned according to ISUP 2014 recommendations [14]. Clinically significant PCa was defined as having met the clinically significant PCa definition according to Epstein's criteria (PSA density $\geq 0.15 \mathrm{ng} / \mathrm{mL} / \mathrm{g}$, Gleason score $\geq 7, \geq 3$ positive cores for $\mathrm{PCa}$, and presence of $\geq 50 \%$ of PCa per any core) [15] or if ISUP grade $\geq 2$ has been identified at biopsy.

2.2. Statistical Analysis. Descriptive statistics was used to characterize patients in groups according to biopsy results. The Shapiro-Wilk test was used to determine the normality of the variables. Student's $t$-test, Wilcoxon singed rank test, and Pearson's chi-squared tests were used for comparisons of continuous and qualitative variables, respectively. The relationship between p2PSA, \%p2PSA, phi, and biopsy Gleason score was evaluated using the Spearman correlation analysis. Before the logistic regression analysis, Spearman correlations were used to check the correlation between the variables. Univariate and multivariate binary logistic regression models have been concluded for the prediction of PCa. The multivariate logistic regression models were fitted using the forward stepwise approach. With the intention to find out if a newer biomarker and its indices can improve the diagnostic accuracy of logistic regression models, p2PSA, \%p2PSA, and phi were added to the base model composed of repeated PB, PV, fPSA, and \%fPSA. The accuracy of the tests was measured by the area under the receiver operating characteristic (ROC) curves (AUC). Odds ratios and $95 \%$ confidence intervals (CI) have been calculated. The specificity at fixed $90 \%$ sensitivity, as well as the best combination of sensitivity and specificity, and the positive (PPV) and negative predictive values (NPV) have been estimated. The determination of cut-off values was based on Youden's index. DeLong et al.'s method has been used to compare the ROC curves [16]. Decision curve analysis (DCA) [17] was used to determine the net benefit of single biomarkers in guiding clinical decision-making on PB. Statistical analysis was performed using SAS version 9.2 (SAS Institute Inc., Carry, NC, USA). $P$ value $<0.05$ was defined as statistically significant.

\section{Results}

PCa has been diagnosed in 112 (53.3\%) out of 210 males enrolled into the study. Clinicopathological characteristics of the study cohort are summarized in Table 1. Clinically significant PCa according to Epstein's criteria and ISUP grade $\geq 2$ have been identified in $81(72.3 \%)$ and $40(35.7 \%)$ out of 112 patients, respectively. Isolated HGPIN at biopsy has been identified in $24(11.4 \%)$ patients. Overall and clinically significant $\mathrm{PCa}$, as well as ISUP $<2 \mathrm{PCa}$, was diagnosed more frequently during the first $\mathrm{PB}$ than in the repeated $\mathrm{PB}$ setting 


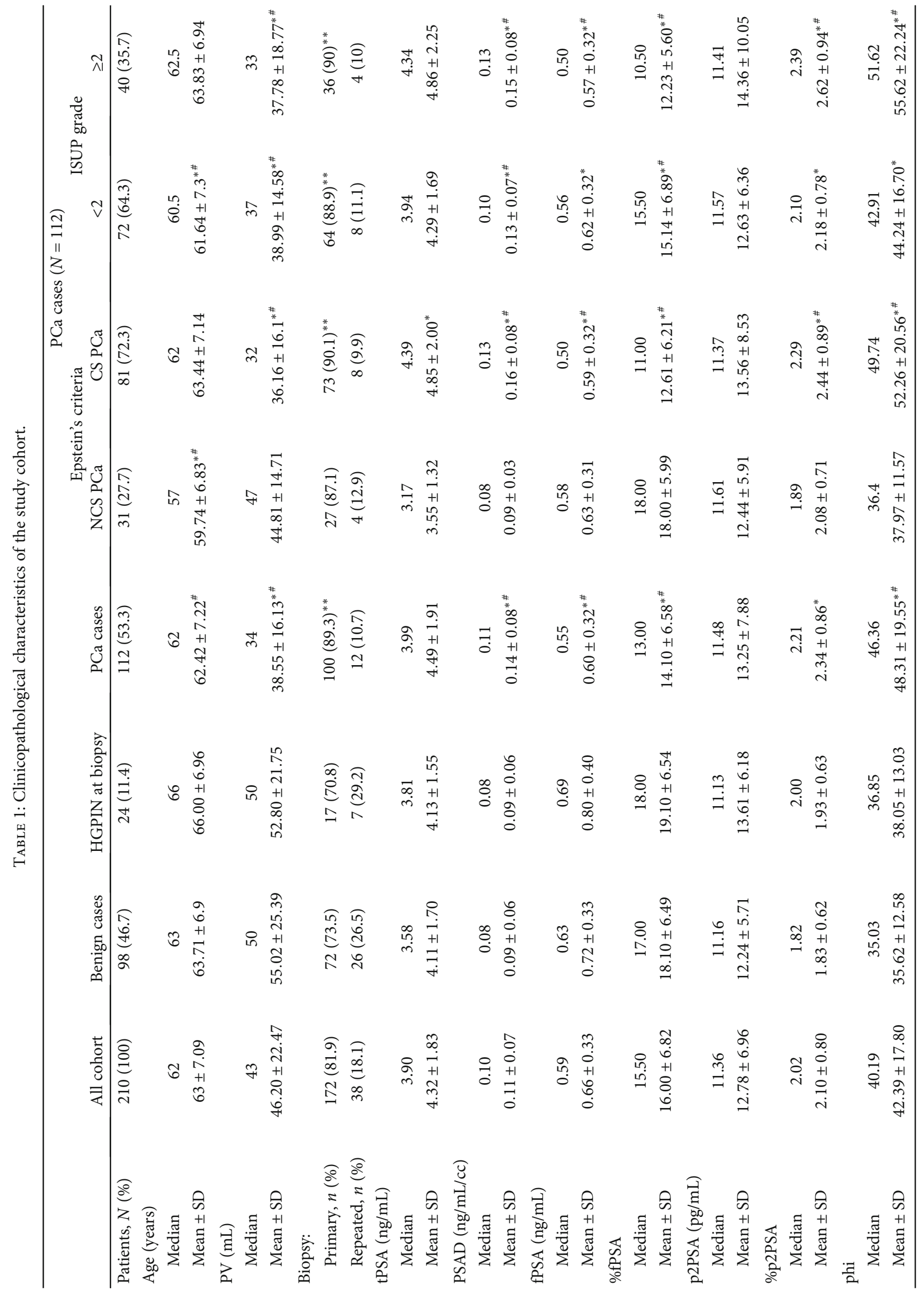




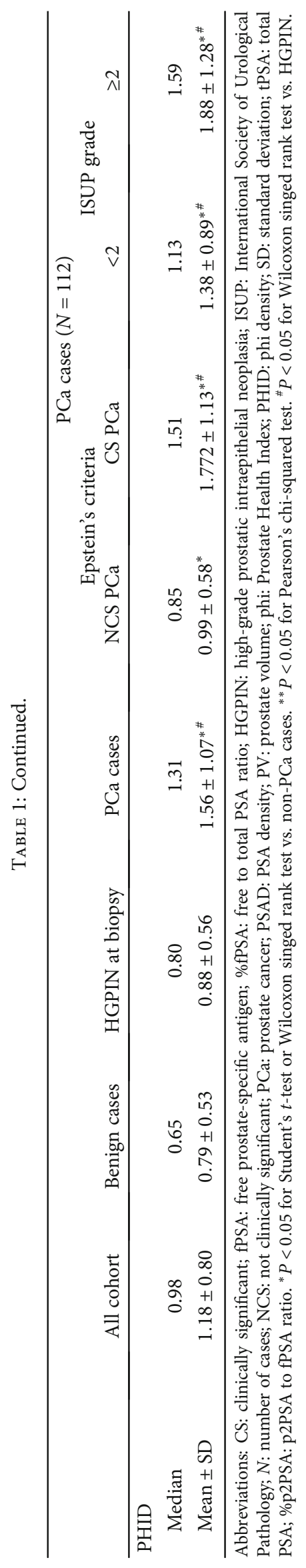




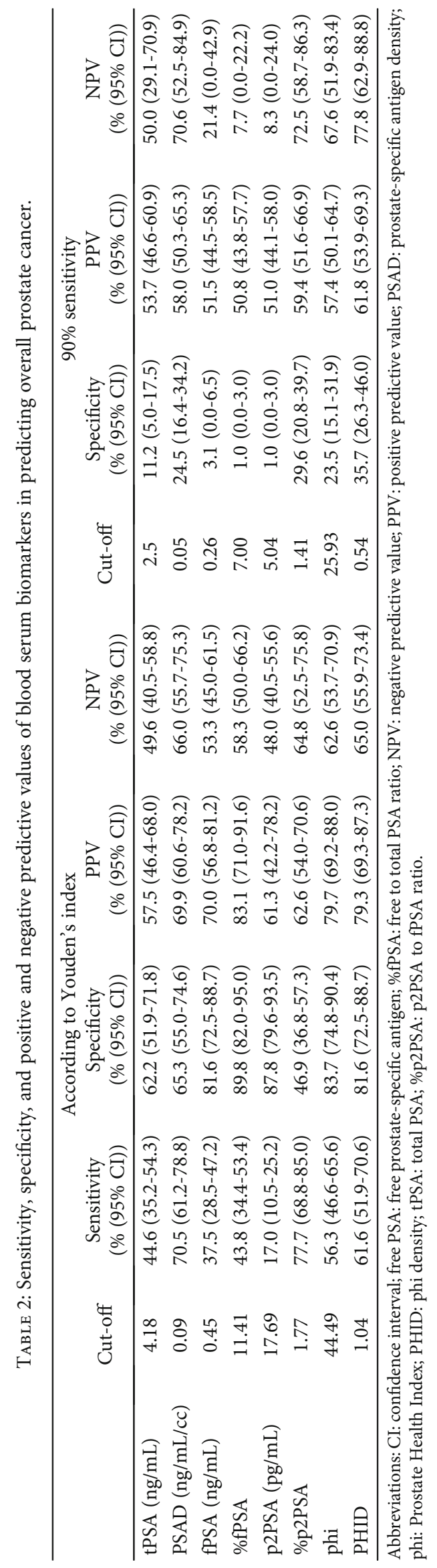




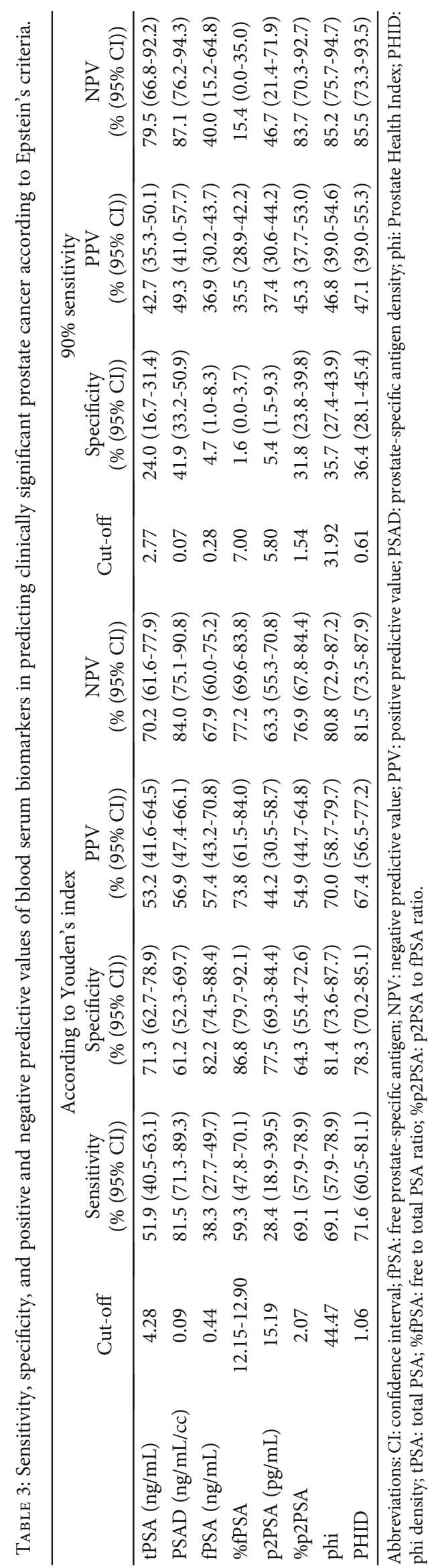




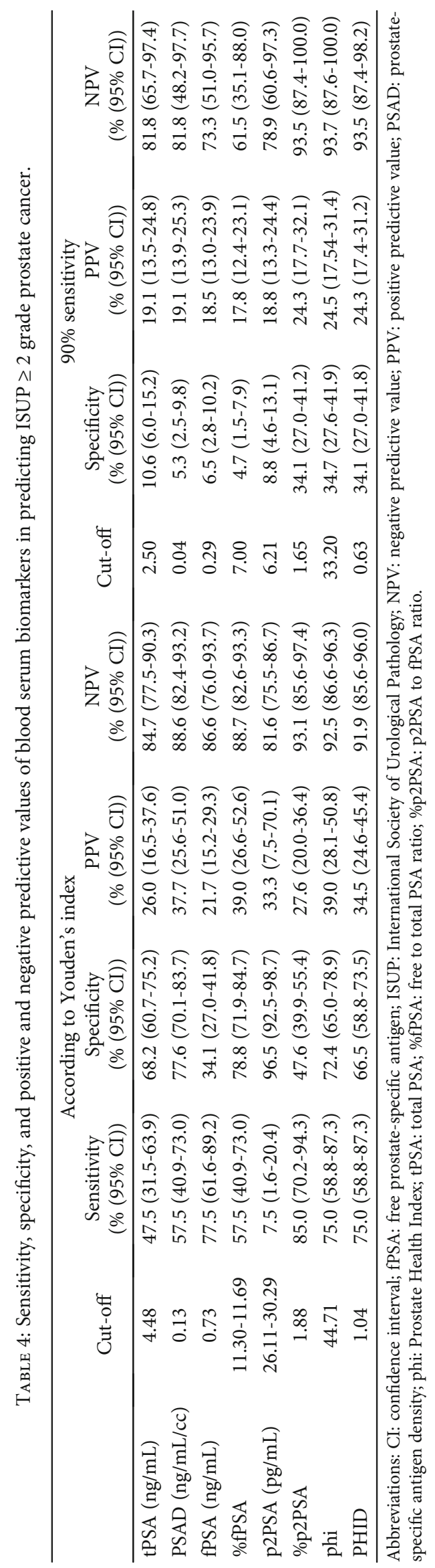


TABLE 5: Area under the receiver operating characteristic curve for prostate cancer biomarkers and logistic regression models.

\begin{tabular}{|c|c|c|c|}
\hline \multirow{4}{*}{ Biomarker } & \multirow{4}{*}{$\begin{array}{c}\text { Overall PCa } \\
\text { AUC (95\% CI) } \\
P \text { value }\end{array}$} & \multicolumn{2}{|c|}{ Clinically significant PCa } \\
\hline & & According to Epstein's criteria & ISUP grade $\geq 2$ \\
\hline & & $\operatorname{AUC}(95 \% \mathrm{CI})$ & AUC $(95 \%$ CI $)$ \\
\hline & & $P$ value & $P$ value \\
\hline \multirow{2}{*}{ tPSA (ng/mL) } & $0.56(0.48-0.64)$ & $0.65(0.57-0.72)$ & $0.58(0.48-0.68)$ \\
\hline & 0.119 & 0.000 & 0.110 \\
\hline \multirow{2}{*}{ PSAD (ng/mL/cc) } & $0.72(0.65-0.79)$ & $0.78(0.72-0.85)$ & $0.68(0.59-0.78)$ \\
\hline & 0.000 & 0.000 & 0.000 \\
\hline \multirow{2}{*}{ fPSA (ng/mL) } & $0.62(0.55-0.70)$ & $0.62(0.54-0.70)$ & $0.62(0.52-0.72)$ \\
\hline & 0.002 & 0.003 & 0.003 \\
\hline \multirow{2}{*}{$\%$ fPSA } & $0.68(0.61-0.75)$ & $0.76(0.69-0.83)$ & $0.71(0.63-0.80)$ \\
\hline & 0.000 & 0.000 & 0.000 \\
\hline \multirow{2}{*}{ p2PSA (pg/mL) } & $0.52(0.44-0.60)$ & $0.52(0.44-0.61)$ & $0.54(0.44-0.64)$ \\
\hline & 0.576 & 0.60 & 0.60 \\
\hline \multirow{2}{*}{$\%$ p2PSA } & $0.68(0.61-0.75)$ & $0.70(0.63-0.77)$ & $0.72(0.64-0.80)$ \\
\hline & 0.000 & 0.000 & 0.000 \\
\hline \multirow{2}{*}{ phi } & $0.72(0.65-0.79)$ & $0.77(0.71-0.84)$ & $0.77(0.69-0.84)$ \\
\hline & 0.000 & 0.000 & 0.000 \\
\hline \multirow{2}{*}{ PHID } & $0.77(0.70-0.83)$ & $0.80(0.74-0.86)$ & $0.75(0.66-0.83)$ \\
\hline & 0.000 & 0.000 & 0.000 \\
\hline \multirow{2}{*}{ Base model } & $0.75(0.69-0.82)$ & $0.81(0.74-0.87)$ & $0.74(0.65-0.83)$ \\
\hline & 0.000 & 0.000 & 0.000 \\
\hline \multirow{2}{*}{ Base model +p2PSA } & $0.78(0.72-0.84)$ & $0.83(0.77-0.89)$ & $0.78(0.70-0.86)$ \\
\hline & 0.000 & 0.000 & 0.000 \\
\hline \multirow{2}{*}{ Base model $+\%$ p 2 PSA } & $0.78(0.71-0.84)$ & $0.82(0.76-0.88)$ & $0.78(0.70-0.86)$ \\
\hline & 0.000 & 0.000 & 0.000 \\
\hline \multirow{2}{*}{ Base model + phi } & $0.78(0.72-0.84)$ & $0.83(0.76-0.89)$ & $0.79(0.71-0.87)$ \\
\hline & 0.000 & 0.000 & 0.000 \\
\hline \multirow{2}{*}{ Base model +PHID } & $0.77(0.71-0.84)$ & $0.80(0.74-0.87)$ & $0.75(0.66-0.83)$ \\
\hline & 0.000 & 0.000 & 0.000 \\
\hline
\end{tabular}

Abbreviations: AUC: area under the receiver operating curve; CI: confidence interval; fPSA: free prostate-specific antigen; \%fPSA: free to total PSA ratio; ISUP: International Society of Urological Pathology; OR: odds ratio; PCa: prostate cancer; \%p2PSA: p2PSA to fPSA ratio; phi: Prostate Health Index; PHID: phi density; PSAD: PSA density; tPSA: total PSA.

( $89.3 \%$ vs. $10.7 \%, 90.1 \%$ vs. $9.9 \%, 90.0 \%$ vs. $10.0 \%$, and $88.9 \%$ vs. $11.1 \%$, respectively; all $P<0.05$; Table 1 ). PV has been found to be significantly smaller in patients harboring overall $\mathrm{PCa}$, as well as in patients with Epstein significant and ISUPgrade $\geq 2 \mathrm{PCa}$ in comparison to patients in the non-PCa group $(38.55 \mathrm{~mL}, 36.16 \mathrm{~mL}$, and $37.78 \mathrm{~mL}$ vs. $55.02 \mathrm{~mL}$, respectively) or to patients with isolated HGPIN at biopsy $(38.55 \mathrm{~mL}$, $36.16 \mathrm{~mL}$, and $37.78 \mathrm{~mL}$ vs. $52.80 \mathrm{~mL}$, respectively; all $P<0.05$; Table 1). tPSA mean value was significantly slightly higher only in patients with Epstein significant PCa in comparison to patients in the non-PCa group $(4.85 \mathrm{ng} / \mathrm{mL}$ vs. $4.11 \mathrm{ng} / \mathrm{mL}$, respectively; $P=0.004$; Table 1 ). PSAD mean value was higher in patients with overall, Epstein significant, and ISUP grade $\geq 2 \mathrm{PCa}$ in comparison to patients in the non-PCa group or with isolated HGPIN at biopsy $(0.14 \mathrm{ng} / \mathrm{mL} / \mathrm{cc}$, $0.16 \mathrm{ng} / \mathrm{mL} / \mathrm{cc}$, and $0.13 \mathrm{ng} / \mathrm{mL} / \mathrm{cc}$ vs. $0.09 \mathrm{ng} / \mathrm{mL} / \mathrm{cc}$, respectively; all $P<0.01$; Table 1$)$.

fPSA and \%fPSA mean values were significantly lower, so phi and PHID mean values were higher in patients with overall, Epstein significant, and ISUP grade $\geq 2$ PCa than in patients in the non-PCa group and isolated HGPIN at biopsy $(0.60 \mathrm{ng} / \mathrm{mL}$,
$0.59 \mathrm{ng} / \mathrm{mL}$, and $0.57 \mathrm{ng} / \mathrm{mL}$ vs. $0.72 \mathrm{ng} / \mathrm{mL}$ and $0.80 \mathrm{ng} / \mathrm{mL}$, so $48.31,52.26$, and 55.62 vs. 35.62 and 38.05, respectively; all $P<0.05$; Table 1). \%p2PSA mean values were higher in patients with overall $\mathrm{PCa}$ in comparison to patients in the non-PCa group (2.34 vs. $1.83, P<0.001)$, as well as in patients with Epstein significant andISUP $\geq 2 \mathrm{PCa}$ in comparison to patients in the non-PCa group and isolated HGPIN at biopsy (2.44 and 2.62 vs. 1.83 and 1.93, respectively; $P<$ 0.05; Table 1).

Significant correlations were revealed between biopsy ISUP grade $\geq 2$ and $\%$ p2PSA $(\rho=0.30, P<0.001)$, phi $(\rho=0.36, P<0.001)$, and PHID $(\rho=0.42, P<0.001)$.

Using Youden's index, PHID with cut-off value of 1.04 for detection of overall PCa, 1.06 for Epstein significant $\mathrm{PCa}$, and 1.04 for ISUP grade $\geq 2 \mathrm{PCa}$ have outperformed tPSA, PSAD, fPSA, \%fPSA, p2PSA, \%p2PSA, and phi and showed the best diagnostic power, with sensitivity and specificity of $61.6 \%$ and $81.6 \%, 71.6 \%$ and $78.3 \%$, and $75.0 \%$ and $66.5 \%$, respectively (Tables $2-4$ ).

At $90 \%$ sensitivity for detecting overall PCa, PHID with cut-off value of 0.54 has had the specificity of $35.7 \%$, which 


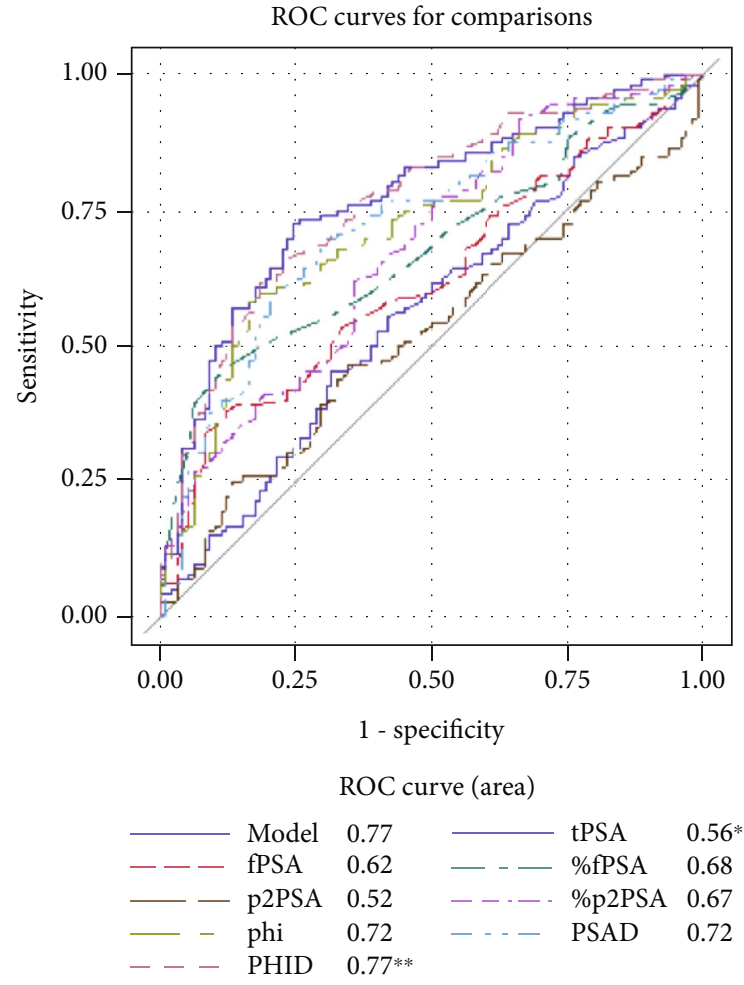

FIGURE 1: Receiver operating characteristic curves representing the diagnostic ability of blood serum biomarkers in predicting overall prostate cancer. Abbreviations: ISUP: International Society of Urological Pathology; fPSA: free prostate-specific antigen; \%fPSA: free to total PSA ratio; phi: Prostate Health Index; PHID: phi density; PSAD: PSA density; ROC: receiver operating characteristic; tPSA: total PSA; \%p2PSA: p2PSA to PPSA ratio. All significant differences are marked with asterisk $\left({ }^{*}\right)$ and $\left({ }^{* *}\right):{ }^{*} P<$ 0.05 for tPSA vs. p2PSA, phi, \%fPSA, and PSAD. ${ }^{* *} P<0.05$ for PHID vs. tPSA, fPSA, \%fPSA, p2PSA, \%p2PSA, and phi.

was higher than other biomarkers. At 90\% sensitivity for detecting Epstein significant PCa, PSAD with cut-off value of $0.07 \mathrm{ng} / \mathrm{mL} / \mathrm{cc}$ has shown the specificity of $41.9 \%$ that was slightly higher than phi and PHID (35.7\% and 36.4\%, respectively). However, at $90 \%$ sensitivity for detecting ISUP grade $\geq 2 \mathrm{PCa}$, phi and PHID with cut-off values of 33.2 and 0.63 have shown the highest specificity of $34.7 \%$ and $34.1 \%$, respectively (Tables $2-4$ ).

At $90 \%$ sensitivity for detecting overall PCa, PHID would lead to avoid $21.4 \%$ of prostate biopsies in comparison to $16.2 \%$ for phi and PSAD, $19 \%$ for $\%$ p2PSA, $10.5 \%$ for tPSA, and less than $7 \%$ for the rest of the biomarkers. At $90 \%$ sensitivity for detecting ISUP grade $\geq 2 \mathrm{PCa}$, phi and PHID, as well as PSAD for detecting Epstein significant PCa, would lead to avoid $30 \%$ of prostate biopsies.

In univariate ROC analysis (Table 5), PHID with AUC of 0.77 was the most accurate predictor of overall PCa significantly outperforming tPSA, fPSA, \%fPSA, p2PSA, \%p2PSA, and phi (all $P<0.05$; see Figure 1). PHID was the most accurate predictor of Epstein significant $\mathrm{PCa}$ with AUC of 0.80 outperforming tPSA, fPSA, p2PSA, and \%p2PSA (all $P<$ 0.05 ; see Figure 2). However, phi was the most accurate predictor of ISUP grade $\geq 2 \mathrm{PCa}$ at biopsy with AUC of

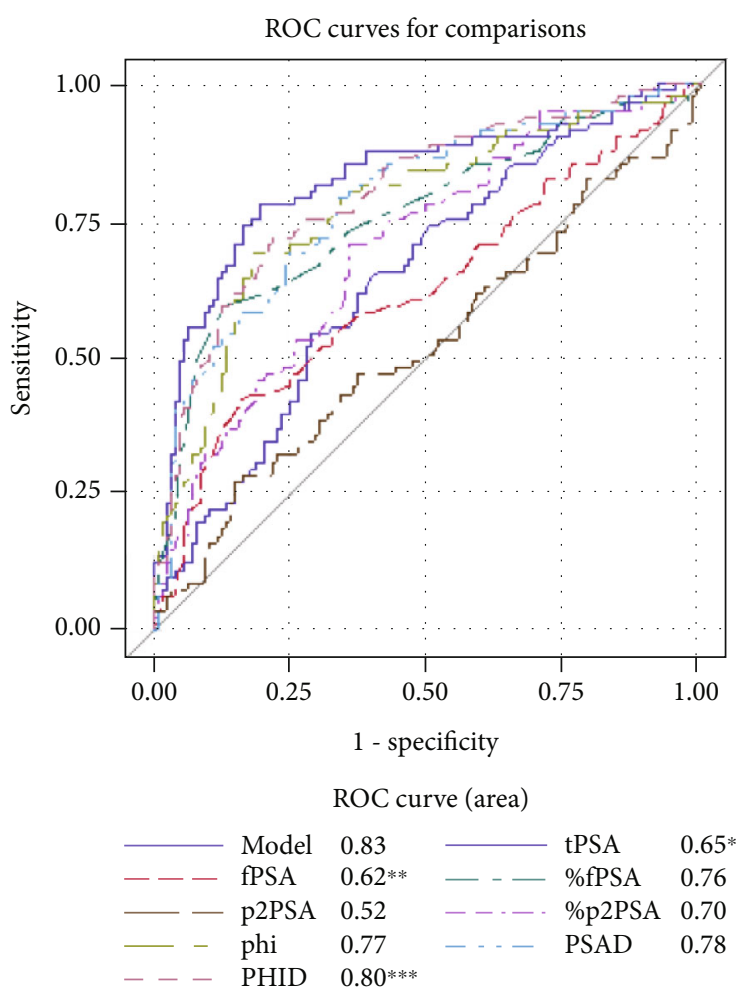

FIGURE 2: Receiver operating characteristic curves representing the diagnostic ability of blood serum biomarkers in predicting clinically significant prostate cancer according to Epstein's criteria. Abbreviations: ISUP: International Society of Urological Pathology; fPSA: free prostate-specific antigen; \%fPSA: free to total PSA ratio; phi: Prostate Health Index; PHID: phi density; PSAD: PSA density; ROC: receiver operating characteristic; tPSA: total PSA; \%p2PSA: p2PSA to fPSA ratio. All significant differences are marked with asterisk $\left({ }^{*}\right),\left({ }^{* *}\right)$, and $\left({ }^{* * *}\right):{ }^{*} P<0.05$ for tPSA vs. \%fPSA, p2PSA, phi, PSAD, and PHID. ${ }^{* *} P<0.05$ for fPSA vs. \%fPSA, phi, PSAD, and PHID. ${ }^{* * *} P<0.05$ for PHID vs. p2PSA and \%p2PSA.

0.77 significantly outperforming tPSA and fPSA (all $P<$ 0.05; see Figure 3).

In multivariate logistic regression analysis by adding p2PSA and its derivatives one by one to the base logistic regression model, which consisted of repeated biopsy, PV, fPSA, and \%fPSA variables, it has been estimated that PHID is the most significant predictor for overall $\mathrm{PCa}$ (OR $4.34, P<0.001$ ), Epstein significant $\mathrm{PCa}(\mathrm{OR} 3.58, P<0.001$ ), and ISUPgrade $\geq 2 \mathrm{PCa}$ (OR $2.38, P<0.001$ ). In all multivariate logistic regression model analysis, p2PSA, \%p2PSA, phi, and PHID have achieved an independent predictor status. The only phi added to the base multivariate logistic regression model significantly improved diagnostic accuracy by $5 \%$ in predicting ISUP grade $\geq 2 \mathrm{PCa}$ at biopsy (AUC 0.74 and 0.79 , respectively; $P=0.039$; Table 6).

$-.09 \mathrm{pt}$ ? $>$ We performed DCA to determine the net benefit for each biomarker in predicting overall and clinically significant PCa. The best net benefit was determined for PHID in predicting overall and Epstein significant $\mathrm{PCa}$ (Figures 4(a) and 4(b)) and for phi in predicting ISUP grade $\geq 2 \mathrm{PCa}$ at biopsy (Figure $4(\mathrm{c})$ ). At $20 \%$ threshold 


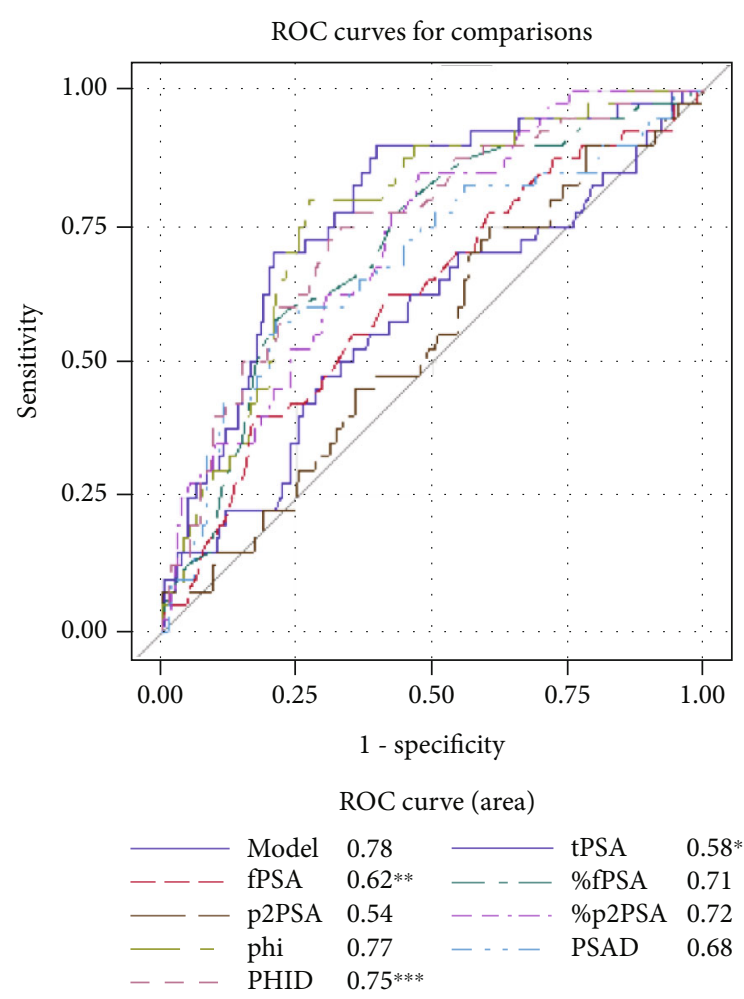

FIGURE 3: Receiver operating characteristic curves representing the diagnostic ability of blood serum biomarkers in predicting ISUP grade $\geq 2$ prostate cancer. Abbreviations: ISUP: International Society of Urological Pathology; fPSA: free prostate-specific antigen; \%fPSA: free to total PSA ratio; phi: Prostate Health Index; PHID: phi density; PSAD: PSA density; ROC: receiver operating characteristic; tPSA: total PSA; \%p2PSA: p2PSA to fPSA ratio. All significant differences are marked with asterisk $\left({ }^{*}\right),\left({ }^{* *}\right)$, and $\left({ }^{* * *}\right)$ : ${ }^{*} P<0.05$ for tPSA vs. $\%$ fPSA, phi, PSAD, and PHID. ${ }^{* *} P<0.05$ for fPSA vs. \%fPSA, \%p2PSA, phi, and PHID. ${ }^{* * *} P<0.05$ for PHID vs. p2PSA and PSAD.

probability, based on PHID, 45 and 26 of 100 biopsied patients would be diagnosed overall and Epstein significant $\mathrm{PCa}$, respectively, so based on phi,ISUP $\geq 2 \mathrm{PCa}$ would be diagnosed in 9 of 100 biopsied males.

\section{Discussion}

In today's clinical practice, there is no universal definition of clinically significant PCa. According to our results, clinically significant PCa according to Epstein's criteria have been diagnosed to $72 \%$ of patients and PCa harboring ISUP grade $\geq 2$ to $36 \%$ of patients. Therefore, the decision to perform PB based on a single serum biomarker with intent to detect clinically significant disease is still a challenge in urological practice.

Due to the limited specificity of tPSA, there is considerable interest in new diagnostic biomarkers for PCa that could overcome tPSA limitations and demonstrate improved specificity. It was found that precursor forms of PSA constitute the predominant fraction of PPSA in PCa serum [18]. Histological analyses of prostate specimens have shown that primarily precursor of PSA, called p2PSA, is elevated in the peripheral zone, while it was undetectable in the transition zone, leading to the consensus that this isoform is more cancer specific than tPSA [19]. Subsequently, it was found that p2PSA isoform is providing higher concentration levels in PCa patients' blood serum [20]. Recently, it was also revealed that p2PSA could be a marker for PCa aggressiveness already several years before diagnosis [21]. So p2PSA derivatives, such as phi and \%p2PSA, have been suggested for PCa diagnostics with intent to increase the specificity of tPSA [13]. Indeed, our study has demonstrated that \%p2PSA and phi are associated with ISUP grade $\geq 2$ disease and could be used for PCa detection, which was also confirmed by other authors $[8,22,23]$. It is reported that \%p2PSA and phi mean values are significantly higher not only in PCa patients in comparison to non-PCa patients, but the difference is found between PCa patients and patients with isolated HGPIN at biopsy [6]. In our study, isolated HGPIN at biopsy have been identified in $11.4 \%$ of patients, and likewise noted above, we determined significantly higher not only \%p2PSA and phi but also PHID mean values in patients with overall, Epstein significant, and ISUP grade $\geq 2 \mathrm{PCa}$ in comparison with patients with isolated HGPIN at biopsy (Table 1).

We estimated that higher PHID mean values are associated not only with overall but also with clinically significant PCa (Table 1), which is consistent with previous studies $[24,25]$.

According to our study results, the specificity of $35.7 \%$ at 90\% sensitivity demonstrated the advantages for PHID at cut-off value of 0.54 in comparison with all other investigated biomarkers for overall PCa detection (Table 2). Our results are consistent with previous studies, when PHID at a cutoff of 0.49 and 0.43 at $90.7 \%$ and $97.9 \%$ sensitivity, respectively, demonstrated the specificity of $30 \%$ and $38 \%$ for detection of overall and clinically significant $\mathrm{PCa}$ (i.e., ISUP grade $\geq 2 \mathrm{PCa}$ or Gleason score $3+3$ cancer detected in $>2$ cores or $>50 \%$ of any one core) $[24,25]$.

At $90 \%$ sensitivity, to detect Epstein significant $\mathrm{PCa}$, phi with cut-off of 31.92 and PHID with cut-off of 0.61 have shown the specificity of $35.7 \%$ and $36.4 \%$, respectively, which was slightly inferior to PSAD with cut-off of $0.07 \mathrm{ng} / \mathrm{mL} / \mathrm{cc}$ and specificity of $41.9 \%$ (Table 3 ). However, at $90 \%$ sensitivity, to detect ISUP grade $\geq 2 \mathrm{PCa}$, phi and PHID with cut-off values of 33.2 and 0.63 have shown the highest specificity of $34.7 \%$ and $34.1 \%$, respectively (Table 4 ). According to the literature, the specificity between $29.7 \%$ and $45.2 \%$ at $90 \%$ sensitivity for phi outperformed the specificity of tPSA $(7.8-26.4 \%)$ and \%fPSA (28.5\%) to detect ISUP grade $\geq 2$ PCa $[8,10,26,27]$.

At $90 \%$ sensitivity, for detecting overall $\mathrm{PCa}, \mathrm{PHID}$ would lead to avoid $21.4 \%$ of prostate biopsies in comparison to $16.2 \%$ for phi and PSAD, $19 \%$ for $\%$ p2PSA, $10.5 \%$ for tPSA, and less than $7 \%$ for the rest of the biomarkers. At $90 \%$ sensitivity for detecting ISUP grade $\geq 2 \mathrm{PCa}$, phi and PHID, as well as PSAD for detecting Epstein significant PCa, would lead to avoid $30 \%$ of prostate biopsies.

On univariate ROC analysis, we have identified PHID as a more accurate predictor for overall PCa detection in comparison to tPSA, fPSA, \%PSA, p2PSA, \%p2PSA, and phi (all $P<0.05$; see Figure 1). What is more important, we came to a conclusion that PHID is the most accurate predictor of 


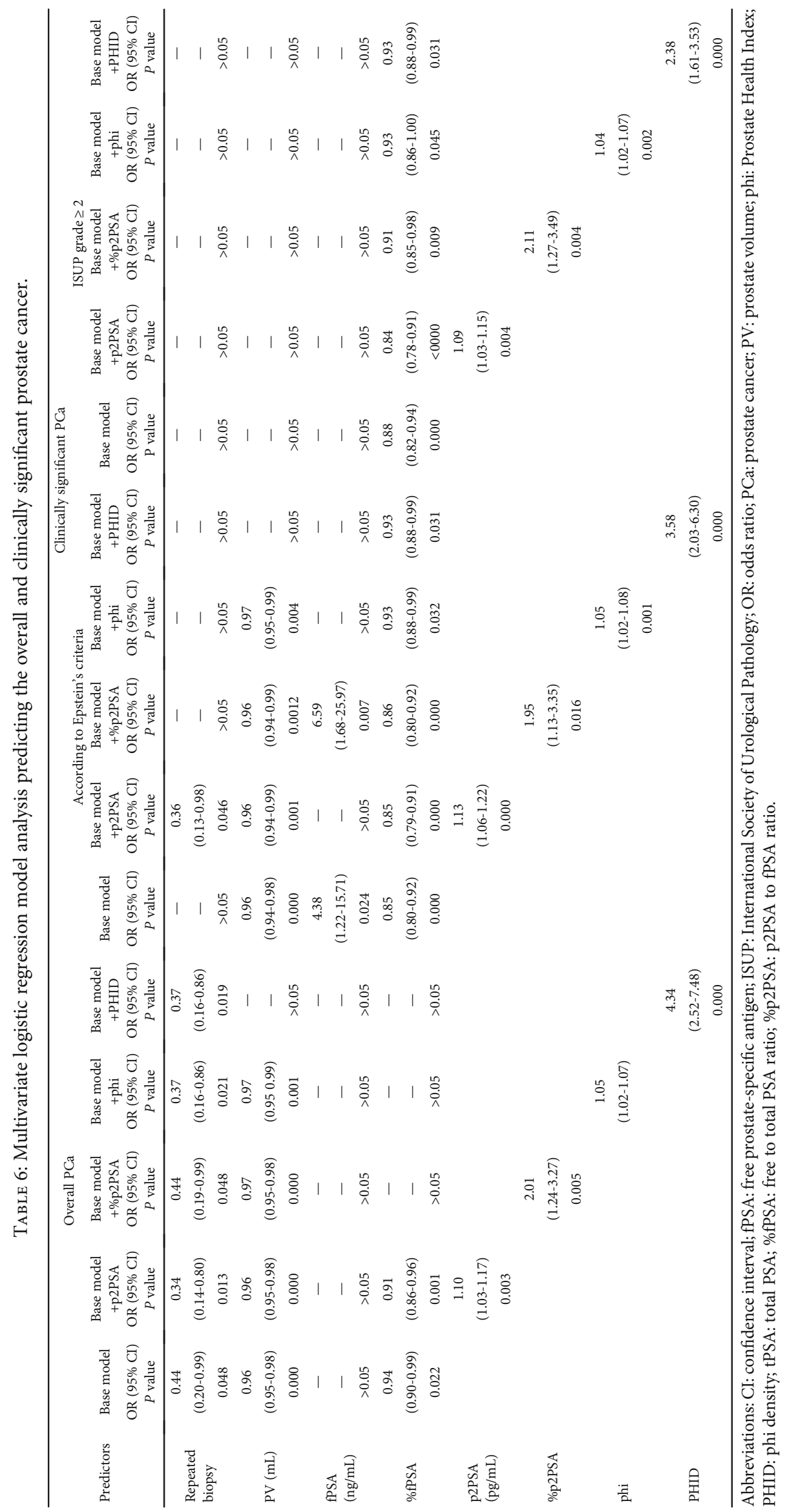




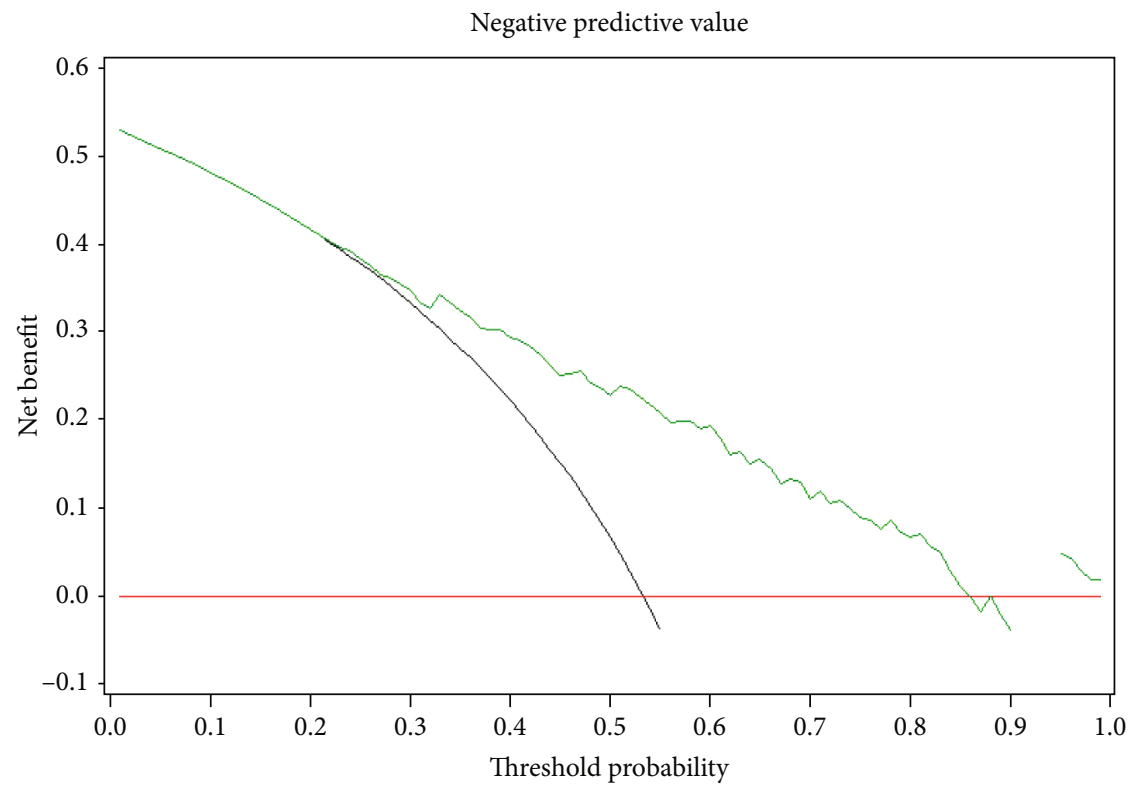

_ Net benefit: treat all

_ Net benefit: treat none

— Net benefit: PHID

(a)

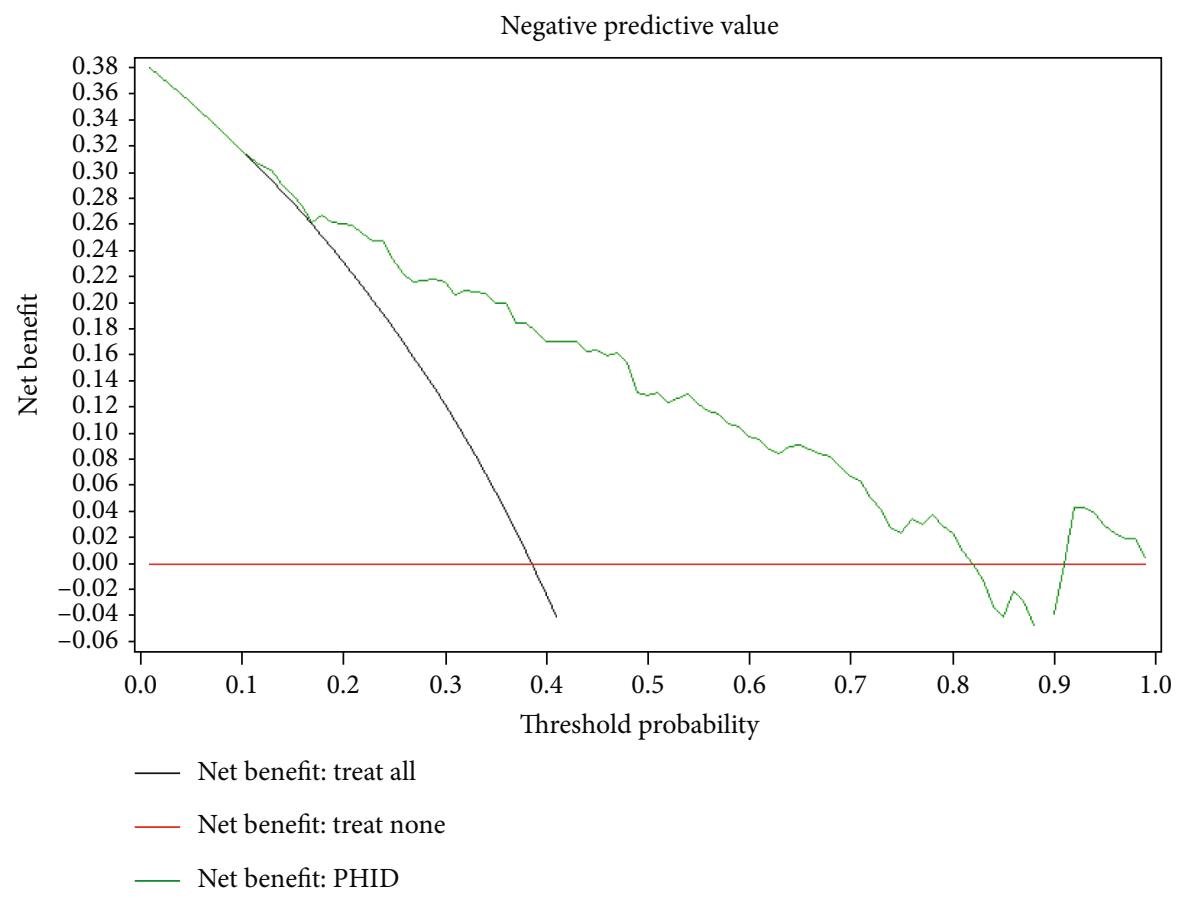

(b)

Figure 4: Continued. 


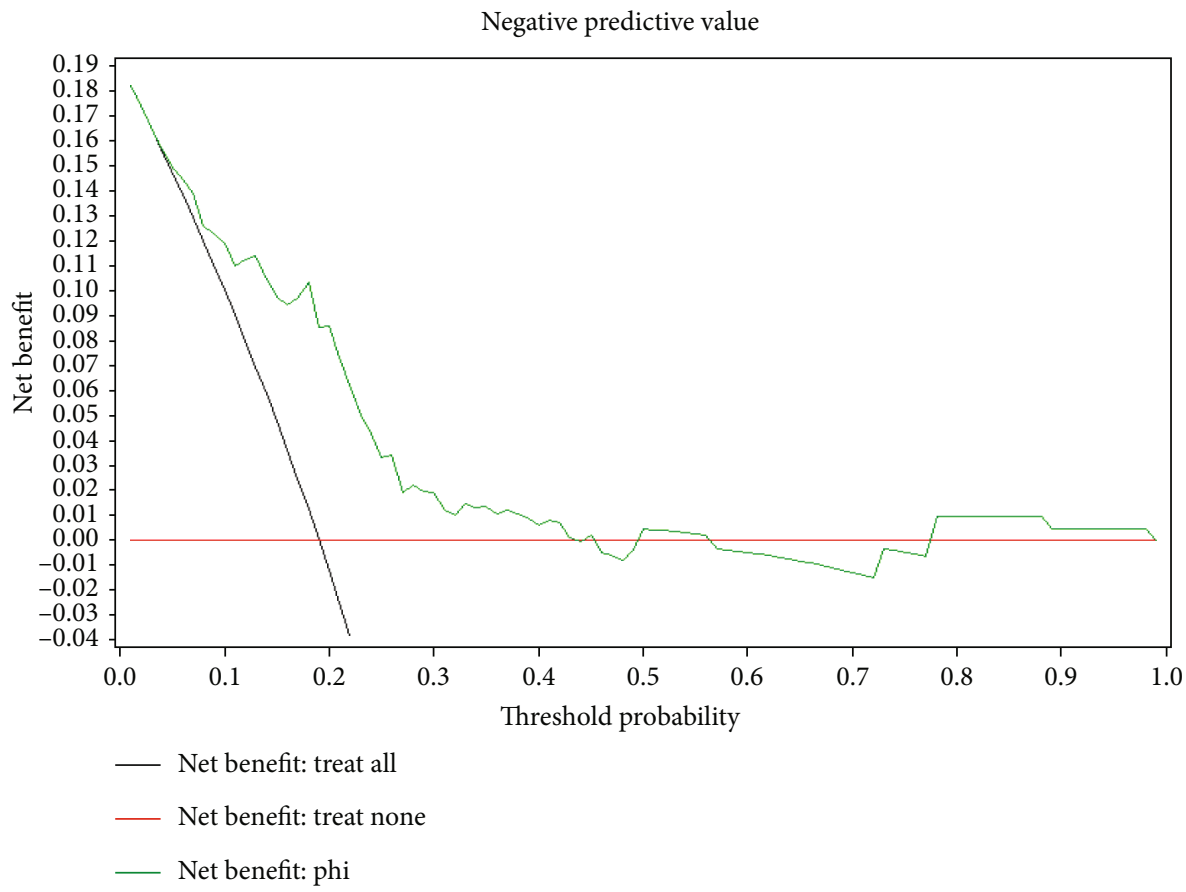

(c)

FIGURE 4: Decision curve analysis for prostate cancer biomarkers that have demonstrated the best net benefit (a) for prediction of overall, (b) for prediction of Epstein significant, and (c) for prediction of ISUP $\geq 2$ prostate cancer. The net benefit is plotted against the threshold probability. The unit of net benefit is true positives. Abbreviations: phi: Prostate Health Index; PHID: phi density.

Epstein significant PCa with AUC of 0.80 outperforming tPSA, fPSA, p2PSA, and \%p2PSA (all $P<0.05$; see Table 2 ). However, phi was the most accurate predictor of ISUP grade $\geq 2 \mathrm{PCa}$ at biopsy with AUC of 0.77 significantly outperforming tPSA and fPSA (all $P<0.05$; see Figure 3 ). Other authors have reported results that are in agreement with our findings, where PHID significantly outperformed tPSA, fPSA, and \% fPSA in prediction for overall PCa, so PHID and phi had the greatest predictive accuracy for clinically significant prostate cancer $[25,26]$.

However, there is no ideal single biomarker and a multivariable approach for improved PCa detection is advocated [28]. The multivariate logistic regression models for PCa prediction, which include $\mathrm{p} 2 \mathrm{PSA}$ and its derivatives, are described in many clinical studies. It was estimated that addition of p2PSA derivatives in a multivariate logistic regression model, which consisted of the most common demographic and clinical PCa predictors, has improved predictive accuracy for overall PCa detection up to $11 \%$ and outperformed its independent components $[22,23,29,30]$. Recently, Loeb et al. came to a conclusion that inclusion of phi into the multivariate logistic regression model, which consisted of age, previous biopsy, PV, and tPSA, improved AUC from 0.70 to 0.75 to predict ISUP grade $\geq 2 \mathrm{PCa}$ in males with negative DRE and PSA between 2 and $10 \mathrm{ng} / \mathrm{mL}$ [31]. In our study, we have revealed that only phi inclusion into the multivariate logistic regression model, which consisted of previous biopsy, PV, fPSA, and $\%$ fPSA, has improved AUC to predict ISUP grade $\geq 2 \mathrm{PCa}$ from 0.74 to $0.79(P=0.04)$.

Summarizing the available scientific data, it is concluded that phi and PHID could help to improve individual risk assessment for early particularly clinically significant PCa detection, to reduce unnecessary biopsies, either may help to select patients eligible for active surveillance and may play a role in treatment decision-making [32].

Nevertheless, we should address several limitations of the present study. Firstly, a small study cohort could lead to inability to establish cut-off values that could be useful in daily practice. Secondly, it was not possible to make a comparative analysis with other commercially available blood biomarkers, including $4 \mathrm{~K}$ test and PCA3, which could be useful tools in making decisions on PB. Thirdly, multiparametric magnetic resonance imaging, widely used nowadays in clinical practice, was not included in our protocol. Finally, several dedicated pathologists have been involved which could make a bias in pathological analysis.

In spite of these limitations, there is a certain strength in our study. This is a prospective study in which all males underwent $\mathrm{PB}$ under a standardized protocol. The study population consisted of males with tPSA levels within the "grey" zone and negative DRE, representing the most debatable group of population in making decisions on $\mathrm{PB}$.

\section{Conclusions}

PHID and phi have shown the greatest specificity at $90 \%$ sensitivity in predicting overall and ISUP grade $\geq 2 \mathrm{PCa}$. phi and PHID have shown slightly inferior specificity at $90 \%$ sensitivity in comparison with PSAD in predicting Epstein significant PCa. phi and PHID, as well as PSAD, could lead to avoid $30 \%$ of unnecessary prostate biopsies in everyday clinical practice. PHID is the most accurate predictor of overall 
and Epstein significant PCa, so phi is the most accurate predictor of ISUP grade $\geq 2$ PCa. phi significantly improves the diagnostic accuracy of multivariable models in predicting ISUP grade $\geq 2 \mathrm{PCa}$ at biopsy.

\section{Data Availability}

The data used to support the findings of this study are available from the corresponding author upon request.

\section{Conflicts of Interest}

The authors declare that there is no conflict of interest regarding the publication of this paper.

\section{Acknowledgments}

We would like to thank Beckman Coulter Lithuania for providing Access Hybritech p2PSA reagents and Immunoassay System Analyzer.

\section{References}

[1] F. Bray, J. Ferlay, I. Soerjomataram, R. L. Siegel, L. A. Torre, and A. Jemal, "Global cancer statistics 2018: GLOBOCAN estimates of incidence and mortality worldwide for 36 cancers in 185 countries," CA: a cancer journal for clinicians, vol. 68, no. 6, pp. 394-424, 2018.

[2] J. Ferlay, I. Soerjomataram, R. Dikshit et al., "Cancer incidence and mortality worldwide: sources, methods and major patterns in GLOBOCAN 2012," International journal of cancer, vol. 136, no. 5, pp. E359-E386, 2015.

[3] W. J. R. J. Catalona, F. R. Ahmann, M. A. Hudson et al., "Comparison of digital rectal examination and serum prostate specific antigen in the early detection of prostate cancer: results of a multicenter clinical trial of 6,630 men," The Journal of Urology, vol. 151, no. 5, pp. 1283-1290, 1994.

[4] I. M. Thompson, D. K. Pauler, P. J. Goodman et al., "Prevalence of prostate cancer among men with a prostate-specific antigen level $\leq 4.0$ ng per milliliter," The New England journal of medicine, vol. 350, no. 22, pp. 2239-2246, 2004.

[5] J. I. Epstein and M. Herawi, "Prostate needle biopsies containing prostatic intraepithelial neoplasia or atypical foci suspicious for carcinoma: implications for patient care," The Journal of urology, vol. 175, no. 3, pp. 820-834, 2006.

[6] M. Ferro, D. Bruzzese, S. Perdonà et al., "Predicting prostate biopsy outcome: prostate health index (phi) and prostate cancer antigen 3 (PCA3) are useful biomarkers," Clinica chimica acta; international journal of clinical chemistry, vol. 413, no. 15-16, pp. 1274-1278, 2012.

[7] E. A. Heijnsdijk, A. der Kinderen, E. M. Wever, G. Draisma, M. J. Roobol, and H. J. de Koning, "Overdetection, overtreatment and costs in prostate-specific antigen screening for prostate cancer," British journal of cancer, vol. 101, no. 11, pp. 1833-1838, 2009.

[8] W. J. Catalona, A. W. Partin, M. G. Sanda et al., "A multicenter study of [-2] pro-prostate specific antigen combined with prostate specific antigen and free prostate specific antigen for prostate cancer detection in the 2.0 to $10.0 \mathrm{ng} / \mathrm{ml}$ prostate specific antigen range," The Journal of urology, vol. 185, no. 5, pp. 1650-1655, 2011.
[9] D. Bruzzese, C. Mazzarella, M. Ferro et al., "Prostate health index vs percent free prostate-specific antigen for prostate cancer detection in men with "gray" prostate-specific antigen levels at first biopsy: systematic review and meta-analysis," Translational research: the journal of laboratory and clinical medicine, vol. 164, no. 6, pp. 444-451, 2014.

[10] C. de la Calle, D. Patil, J. T. Wei et al., "Multicenter evaluation of the prostate health index to detect aggressive prostate cancer in biopsy naïve men," The Journal of urology, vol. 194, no. 1, pp. 65-72, 2015.

[11] M. Boegemann, C. Stephan, H. Cammann et al., "The percentage of prostate-specific antigen (PSA) isoform [-2]proPSA and the Prostate Health Index improve the diagnostic accuracy for clinically relevant prostate cancer at initial and repeat biopsy compared with total PSA and percentage free PSA in men aged $\leq 65$ years," BJU international, vol. 117, no. 1, pp. 72-79, 2016.

[12] A. Semjonow, T. Kopke, E. Eltze, B. Pepping-Schefers, H. Burgel, and C. Darte, "Pre-analytical in-vitro stability of [-2]proPSA in blood and serum," Clinical Biochemistry, vol. 43, no. 10-11, pp. 926-928, 2010.

[13] F. H. Jansen, R. H. N. van Schaik, J. Kurstjens et al., "Prostatespecific antigen (PSA) isoform p2PSA in combination with total PSA and free PSA improves diagnostic accuracy in prostate cancer detection," European urology, vol. 57, no. 6, pp. 921-927, 2010.

[14] J. I. Epstein, L. Egevad, M. B. Amin et al., "The 2014 International Society of Urological Pathology (ISUP) consensus conference on Gleason grading of prostatic carcinoma: definition of grading patterns and proposal for a new grading system," The American journal of surgical pathology, vol. 40, no. 2, pp. 244-252, 2016.

[15] J. I. Epstein, P. C. Walsh, M. Carmichael, and C. B. Brendler, "Pathologic and clinical findings to predict tumor extent of nonpalpable (stage T1c) prostate cancer," Journal of the American Medical Association, vol. 271, no. 5, pp. 368-374, 1994.

[16] E. R. DeLong, D. M. DeLong, and D. L. Clarke-Pearson, "Comparing the areas under two or more correlated receiver operating characteristic curves: a nonparametric approach," Biometrics, vol. 44, no. 3, pp. 837-845, 1988.

[17] A. J. Vickers and E. B. Elkin, "Decision curve analysis: a novel method for evaluating prediction models," Medical decision making: an international journal of the Society for Medical Decision Making, vol. 26, no. 6, pp. 565-574, 2016.

[18] S. D. Mikolajczyk, L. S. Grauer, L. S. Millar et al., "A precursor form of PSA (pPSA) is a component of the free PSA in prostate cancer serum," Urology, vol. 50, no. 5, pp. 710-714, 1997.

[19] S. D. Mikolajczyk, L. S. Millar, T. J. Wang et al., "A precursor form of prostate-specific antigen is more highly elevated in prostate cancer compared with benign transition zone prostate tissue," Cancer research, vol. 60, no. 3, pp. 756759, 2000.

[20] S. D. Mikolajczyk, K. M. Marker, L. S. Millar et al., “A truncated precursor form of prostate-specific antigen is a more specific serum marker of prostate cancer," Cancer research, vol. 61, no. 18, pp. 6958-6963, 2001.

[21] I. Heidegger, H. Klocker, E. Steiner et al., "[-2]proPSA is an early marker for prostate cancer aggressiveness," Prostate cancer and prostatic diseases, vol. 17, no. 1, pp. 70-74, 2014.

[22] G. Guazzoni, L. Nava, M. Lazzeri et al., "Prostate-specific antigen (PSA) isoform p2PSA significantly improves the prediction of prostate cancer at initial extended prostate biopsies in patients with total PSA between 2.0 and $10 \mathrm{ng} / \mathrm{ml}$ : results of 
a prospective study in a clinical setting," European urology, vol. 60, no. 2, pp. 214-222, 2011.

[23] H. Park, S. W. Lee, G. Song et al., "Diagnostic performance of $\%[-2]$ proPSA and prostate health index for prostate cancer: prospective, multi-institutional study," Journal of Korean medical science, vol. 33, no. 11, article e94, 2018.

[24] L. Mearini, C. Ferri, M. Lazzeri et al., "Evaluation of prostatespecific antigen isoform p2PSA and its derivates, \%p2PSA, prostate health index and prostate dimension-adjusted related index in the detection of prostate cancer at first biopsy: an exploratory, prospective study," Urologia internationalis, vol. 93, no. 2, pp. 135-145, 2014.

[25] J. J. Tosoian, S. C. Druskin, D. Andreas et al., "Prostate health index density improves detection of clinically significant prostate cancer," BJU international, vol. 120, no. 6, pp. 793-798, 2017.

[26] S. Loeb, M. G. Sanda, D. L. Broyles et al., "The prostate health index selectively identifies clinically significant prostate cancer," The Journal of urology, vol. 193, no. 4, pp. 1163-1169, 2015.

[27] C. Stephan, S. Vincendeau, A. Houlgatte, H. Cammann, K. Jung, and A. Semjonow, "Multicenter evaluation of [-2] proprostate-specific antigen and the prostate health index for detecting prostate cancer," Clinical chemistry, vol. 59, no. 1 , pp. 306-314, 2013.

[28] D. G. Murphy, T. Ahlering, W. J. Catalona et al., "The Melbourne consensus statement on the early detection of prostate cancer," BJU international, vol. 113, no. 2, pp. 186-188, 2014.

[29] L. J. Sokoll, M. G. Sanda, Z. Feng et al., "A prospective, multicenter, National Cancer Institute Early Detection Research Network study of [-2]proPSA: improving prostate cancer detection and correlating with cancer aggressiveness," Cancer epidemiology, biomarkers \& prevention, vol. 19, no. 5, pp. 1193-1200, 2010.

[30] Y. T. Cheng, C. H. Chiang, Y. S. Pu et al., "The application of p2PSA $\%$ and prostate health index in prostate cancer detection: a prospective cohort in a tertiary medical center," Journal of the Formosan Medical Association, vol. 118, no. 1, pp. 260$267,2019$.

[31] S. Loeb, S. S. Shin, D. L. Broyles et al., "Prostate Health Index improves multivariable risk prediction of aggressive prostate cancer," BJU international, vol. 120, no. 1, pp. 61-68, 2017.

[32] M. Ferro, O. de Cobelli, G. Lucarelli et al., "Beyond PSA: the role of prostate health index (phi)," International Journal of Molecular Sciences, vol. 21, no. 4, p. 1184, 2020. 